\title{
A New Approach: Re-Activating Quadriceps After ACL Reconstruction by using Reciprocal Inhibition and Neuro-Muscular Stimulation of Vastus Medialis Oblique
}

\section{Sweta Christian DPT*}

Physical Therapist, New Jersey, USA

*Corresponding Author: Sweta Christian DPT, Physical Therapist, New Jersey, USA.

Received: May 24, 2019; Published: June 01, 2019

DOI: $10.31080 /$ ASOR.2019.02.0059

In my years of working as a physical therapist, I have come across a variety of patients, in different age groups, who have sustained ACL injury. I have also extensively evaluated and treated patients (young athletes and middle aged) who underwent ACL reconstruction surgery using allograft and autograft.

Anterior cruciate ligament (ACL) is a major ligament of the knee complex. Anterior cruciate ligament (ACL) injury is one of the most common type of sports injury that involves sudden, transitional movement of the knee joint, change in direction, jumping and landing sport activities such as soccer, baseball, skiing and football. Patients normally complain of hearing a "pop", when an ACL injury occurs, which is then followed by severe swelling, moderate to complete loss of range of motion, and inability to weight bear on the injured knee.

In my recent and past years, I have treated patients with ACL reconstruction involving allograft and autograft. When treating ACL, quadriceps firing or activation plays a vital role in the recovery following ACL reconstruction. When it comes to the choice of graft, studies have shown that autograft shows rapid improvement and better outcomes than a graft from a cadaver. Furthermore, as autografts are generated from the species own body, the healing response is better with physical therapy. On the contrary, allograft or cadaver graft have shown a higher rate of failure in athletes.

The paradigm shift in treating patients with ACL reconstruction, lies in the insufficiency to fire quadriceps, which is the subsequent cause of decrease ROM and decreased extension at the terminal range. It is necessary to understand that neuromuscular efficiency of quadriceps, especially the VMO (vastus medialis oblique) is one of the major muscle contributing to knee stability.

In my past and recent years, I have used techniques to rapid fire the quadriceps muscle group by using neuromuscular rehabilitation, low-loading aquatic therapy, strengthening exercises and stretching. After utilizing concepts from various techniques,
I have found out that reciprocal inhibition, which is nothing, but a neuromuscular reflex technique, have shown promising results. Reciprocal Inhibition (RI) is a neuromuscular reflex that inhibits the opposite muscle during movement. For e.g, if one contracts the knee extensors (quadriceps), then the opposite group muscles, which are the flexors (hamstrings) are inhibited. Reciprocal inhibition along with Neuromuscular electrical stimulation (NMES) have shown improvement in quadriceps lag in the early stage of ACL repair.

The idea behind using the RI and NMES to activate the VMO, is to use the central nervous system pathway to activate the agonist group (here the quadriceps) to contract, while using the NMES on the VMO. While doing so, the impulses from the antagonist muscle group (hamstring) are carried by the motor neurons to the central nervous system, and thus helping the antagonist muscles to relax.

The reasons that led to this study is: to improve the recovery time in patients with ACL reconstruction surgery, especially athletes who are on limited time constraint and have a need to return back to their sport-related activities.

\section{Volume 2 Issue 7 July 2019}

(C) All rights are reserved by Sweta Christian DPT. 